\title{
Combustion and polymer composites
}

\section{De-Yi Wang PhD}

Head of High Performance Polymer Nanocomposites Group, Madrid Institute for Advanced Studies of Materials (IMDEA Materials), Madrid, Spain

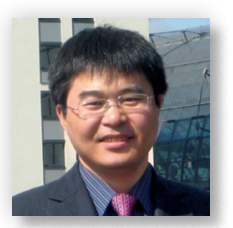

Combustion and flammability are natural for most polymeric materials. Since flammable polymers have been used in various fields in modern society, fire retardancy of polymeric materials has become an important, active and developing field in polymeric materials. However, conventional fire-retardant polymers and additives are facing environmental concerns and stringent fire safety standards and regulations. In recent decades, the dramatic increase in use of halogenated flame retardants (FRs) has had serious impacts on the environment. Several halogenated FRs are determined as persistent organic pollutants by the Stockholm Convention. In some conditions, use of such FRs could produce dioxins and/or furan, which can bioaccumulate significantly and is linked to serious health effects such as cancer. ${ }^{1}$ Consequently, from a chemical and toxicity point of view, the European Union has been proactive in imposing strict restrictions on the use of certain chemical substances; they are being controlled through relatively new legislation such as the restrictions on hazardous substances (RoHS) in electrical and electronic equipment, the waste electrical and electronic equipment (WEEE) directive and the European chemical registration legislation, REACH. Some widely used fire retardants will be phased out soon, while demand for sustainable, environmentally friendly, high-performance fireretardant polymers has increased considerably.

It has been reported that world demand for fire-retardant additives is forecast to rise $6.1 \%$ per year to 2.2 million metric tons in $2014 .^{2}$ In fact, recent years have seen a growing interest in the exploration of new environmentally friendly fire retardants, including bio-based fire retardants, ${ }^{3,4}$ nano-fire retardant ${ }^{5,6}$ and so on, in order to prepare high-performance functional composites. Nevertheless, a significant challenge remains. The nextgeneration FR should have the following qualities: sustainability, environmental friendliness, high efficiency, smoke suppression and multifunctionality.

In an effort to help address these challenges, this themed issue on 'Combustion and polymer composites' compiles papers on novel fire retardants, understanding the combustion behaviours of polymer composites and novel fire-testing approaches for polymer materials and other functional composites. This themed issue aims to show a wide variety of perspectives, from fundamental research to applicative approaches in the fields of fire-retardant materials and functional composites.
In this themed issue, Professor Ping Zhu and his colleagues developed a series of flame-retardant metal alginates. Alginate is one of the most abundant biodegradable and sustainable natural resources in the world, and has been regarded as an alternative to petroleum-based polymers. By studying the combustion behaviours of these functional alginate materials, it has been found that the iron alginate provides high limiting oxygen index (LOI) value $(\mathrm{LOI}=42)$ and passes the UL94 V-0 rating. In comparison, the conventional sodium alginate has a relatively low LOI value and does not achieve UL94 rating. These researches demonstrate the potential of developing new, sustainable, green fire-retardant materials.

This themed issue also contains a contribution from $\mathrm{Mr}$ Bo Wu and his colleagues on a new method to test the combustion properties of molten solid/liquid/powder. They state that a better understanding of the burning behaviour of polymer materials is of great importance to reducing the risks of fire disasters. Their set of tests on PC and PP in different forms showed that the combustion behaviour is quite different for the same sample, and their method provides great insight into this difference. This new method may help people fully and properly understand the combustion behaviours of these materials.

In summary, the study of flame-retardant materials and functional composites is an important research topic. Investigations into fire safety, energy conservation and sustainability, and new fire-retarding techniques - such as functional natural fire retardants, multifunctional nanomaterials and rare earth metal-based materials - all offer promising ways to develop the next generation of fire-retardant polymer materials.

\section{REFERENCES}

1. Bertazzi, P. A.; Consonni, D.; Bachetti, S.; Rubagotti, M.; Baccarelli, M.; Zocchetti, C.; Pesatori, A. C. Health effects of dioxin exposure: a 20-year mortality study. American Journal of Epidemiology 2001, 153, 1031-1044.

2. Freedonia. See http://www.freedoniagroup.com for further details. Accessed 17/03/2014.

3. Wang, X.; Hu, Y.; Song, L.; Xuan, S. Y.; Xing, W. Y.; Bai, Z. M.; Lu, H. D. Flame retardancy and thermal degradation of intumescent flame retardant poly(lactic acid)/starch 
biocomposites. Industrial \& Engineering Chemistry Research 2011, 50, 713-720.

4. Kang, N. J.; Wang, D. Y. A green functional nanohybrid: preparation, characterization and properties of a B-cyclodextrin based functional layered double hydroxide. Journal of Materials Chemistry A 2013, 1, 11376-11383.

5. Costa, F. R.; Wagenknecht, U.; Heinrich, G. LDPE/Mg-Al layered double hydroxide nanocomposite: thermal and flammability properties. Polymer Degradation and Stability 2007, 92, 1813-1823.

6. Wang, D. Y.; Das, A.; Costa, F. R.; Leuteritz, A.; Wang, Y. Z.; Wagenknecht, U.; Heinrich, G. Synthesis of organo cobaltaluminum layered double hydroxide via a novel singlestep self-assembling method and its use as flame retardant nanofiller in PP. Langmuir 2010, 26, 14162-14169. 